\title{
Differential Leukocyte miRNA Responses Following Pan T Cell, Allorecognition and Allosecretome- Based Therapeutics Activation
}

Xining Yang

Terry Fox Research Institute

Wendy M Toyofuku

Canadian Blood Services

Mark D. Scott ( $\square$ mdscott@mail.ubc.ca)

Canadian Blood Services https://orcid.org/0000-0002-2882-156X

\section{Research}

Keywords: T lymphocyte, immunomodulation, miRNA, alloactivation, secretome, tolerance, inflammation, pan $T$ cell activation, therapeutics

Posted Date: July 30th, 2020

DOI: https://doi.org/10.21203/rs.3.rs-48384/v1

License: (c) (i) This work is licensed under a Creative Commons Attribution 4.0 International License. Read Full License 


\section{Abstract}

Background Effective immunomodulation of T cell responses is critical in treating both autoimmune diseases and cancer. Our previous studies have demonstrated that secretomes derived from control or methoxypolyethylene glycol (mPEG) mixed lymphocyte alloactivation assays exerted potent immunomodulatory activity that was mediated by microRNAs (miRNA). In this study, the immunomodulatory effects of biomanufactured miRNA-based allo-secretome therapeutics (SYN, TA1, IA1 and IA2) were compared to Pan T cell activators (PHA and anti-CD3/CD28) and alloactivation (MHCdisparate donors; \pm mPEG grafting). The differential effects of these activation strategies on resting PBMC were assessed via T cell differentiation and proliferation as well as the differential expression of multiple miRNA.

Results Mitogen-induced PBMC proliferation (average of > 85\%) significantly exceed that arising from either allostimulation $(\sim 30 \%)$ or the proinflammatory IA1 secretome product ( 12\%). Consequent to stimulation, the ratio of CD4 to CD8 cells of the resting PBMC (CD4:CD8; 1.7 \pm 0.1 ) decreased in the Pan-T cell, allrecognition and IA1 activated cells (average of $1.1 \pm 0.2 ; 1.2 \pm 0.1$ and $1.0 \pm 0.1$ ). These changes arose consequent to the expansion of both $\mathrm{CD} 4^{+} \mathrm{CD} 8^{+}$and $\mathrm{CD} 4^{-} \mathrm{CD} 8^{-}$populations and the shrinkage of the CD4 subset relative to the expansion of the CD8 T cells. Most importantly, this study demonstrated that these activation strategies exert profoundly unique effects on the differential expression of miRNA within the treated PBMC and that these 'differential patterns of miRNA expression' are associated with significant differences in cellular differentiation and biological function.

Conclusions These findings support the concept that the 'differential pattern of miRNA expression', not a change in a single miRNA, governs the biologic immune response in a 'lock and key' manner. The biomanufacturing of miRNA-enriched secretome biotherapeutics may be a successful approach for producing miRNA cocktails (e.g., TA1 and IA1) that replicate the normal biological 'lock and key'miRNA configuration necessary for the systemic treatment of autoimmune diseases (TA1) or enhancing the endogenous immune response to cancer (IA1).

\section{Background}

Effective modulation of the T cell immune response is critical in treating both autoimmune (e.g., multiple sclerosis, arthritis, type I diabetes) and immunodeficient (e.g., cancer) diseases.[1-4] Treatment of autoimmune disease have commonly focused on cytotoxic agents (e.g., cyclosporine) to remove selfreactive immune cells and more recently, cytokine absorptive approaches (e.g., etanercept).[5-8] These 'anti-inflammatory' approaches have been highly successful in preventing graft rejection and in modulating disease severity in a number of autoimmune diseases. In contrast, in diseases characterized by a weak immune response (e.g., cancer), enhancement of the inflammatory response have attempted to use mitogens (e.g., phytohemagglutinin; PHA), monoclonal antibodies (mAb; e.g., anti-CD3/CD28), cytokines (e.g., IL-2) and, less commonly, induction of an alloresponse (e.g., Coley's toxins and Graft versus Leukemia effects) to strengthen the immune response.[9-18] However, these pro-inflammatory 
approaches have been much less successful due to overly robust responses resulting in adverse events such as cell injury and cytokine release syndrome.

In contrast to these traditional approaches, bioengineering of cell surfaces have also been shown to induce an immunomodulatory effect. The immunocamouflage of the lymphocyte membrane by the covalent grafting of biocompatible polymers (e.g., methoxypolyethylene glycol; mPEG) has been demonstrated to induce a pro-tolerogenic environment both in vitro and in vivo.[19-24] Surprisingly, these studies also found that the secretome from the control and MPEG allorecognition-based mixed lymphocyte reactions (MLR) also exerted potent immunomodulatory activity that was mediated by microRNAs (miRNA). [3, 4, 24, 25]

Mammalian miRNA are short [ 22-nucleotide long] RNA molecules that regulate messenger RNA (mRNA) expression at a posttranscriptional level. Currently, more than 2,000 miRNA have been identified in humans.[26] Since their discovery in 1993 in the nematode $C$. elegans, the role of miRNA has transitioned from being 'junk nucleic acids'to being recognized as key regulators of a multitude of biological processes including the immune response.[27, 28] However, to date, the vast majority of research into the role of miRNA in immune response has been largely observational, with specific miRNA being used as biomarkers of immunological and/or pathogenic disease states.[29, 30] Indeed, the profiles of cellular miRNA expression can actively reflect the systemic alterations in immune activity.[31-33] More recently, due to their rapid response and sensitivity to changing cellular environment, miRNA have also been used as potential biomarkers for drug efficacy prediction and therapeutic approaches.[29]

However, despite their vast potential as biomarkers, relatively little research has been done on miRNA as therapeutic agents largely due to the complexity of miRNA-based bioregulation.[34, 35] It is important to note that miRNA are 'low fidelity' in nature in that a single miRNA can interact with potentially hundreds of genes and a single gene can be regulated by hundreds of miRNA.[36, 37] Hence, replicating the 'pattern of miRNA expression' is key to exerting a desired bioregulatory effect. To reproduce the miRNA patterns necessary to induce either a pro-inflammatory or tolerogenic immune response, our laboratory has utilized an alloresponse-based biomanufacturing approach. In this strategy, alloreaction-derived cell-free secretomes are manufactured from resting peripheral blood mononuclear cells (PBMC) or control- or mPEG-MLR.[3, 4, 24, 25] As previously demonstrated, the miRNA-based secretomes systemically reorientate the immune system towards either a pro-inflammatory (IA1) or pro-tolerogenic (TA1) state.[3, 4, 25] Importantly, miRNA prepared from resting immune cells (human PBMC or murine splenocytes) exhibited minimal immunomodulatory activity.[4, 25]

In this study, we further defined the differential effects of our previously described miRNA-based secretome therapeutic approach $[3,4,25]$ to pan T cell (PHA; anti-CD3/CD28) and allorecognition-based activation of resting human PBMC. T cell differentiation as well as the differential miRNA production induced by the different activation strategies were assessed. Within the miRNA, we further analyzed a panel of thirteen differentially expressed miRNA to associate the miRNA 'pattern of expression' with the PBMC differentiation and biological activity. As will be demonstrated, highly distinct intracellular miRNA 
expression patterns were observed between the pan T cell activators, allorecognition and secretome therapeutics. Indeed, these studies support the view that a 'lock and key' mechanism based on the 'pattern of miRNA expression', rather than changes in single miRNA, underlies the biologic signaling necessary to induce a desired (tolerance versus inflammation) immunomodulatory response.

\section{Results}

miRNA-based secretome therapeutics (SYN, TA1, IA1 and IA2) have been successfully produced in our laboratory based on allorecognition ( \pm polymer-mediated immunocamouflage) based MLR.[3, 4, 25] As demonstrated in our previous studies, the effects of our acellular secretome products to pan T cell (PHA or anti-CD3/CD28) on $\mathrm{CD}^{+} \mathrm{T}$ cell proliferation (Fig. 2, center circle) as well as $\mathrm{T}$ regulatory $\left(\mathrm{CD} 4^{+}{ }^{\mathrm{Foxp}} 3^{+} \mathrm{CD} 25^{+}\right)$and Th17 $\left(\mathrm{CD} 4^{+} \mathrm{IL} 17^{+}\right)$cells were distinct. $[3,4,25]$ To further define the proliferation pattern within the $\mathrm{CD} 3^{+}$cells, $C D 4^{ \pm}$and $\mathrm{CD} 8^{ \pm}$subsets and the $\mathrm{CD}: \mathrm{CD} 8$ ratio were further analyzed (Fig. 2). As shown, resting PBMC demonstrated minimal proliferation and a CD4:CD8 ratio of $1.7 \pm 0.1$. In contrast, the pan $T$ cell activators anti-CD3/CD28 and PHA induced massive $C D 3^{+}$cell proliferation and altered the CD4:CD8 ratio (Fig. 2A). Despite both PHA and anti-CD3/CD28 being Pan T cell activators, there were differences in how these agents modulated the CD4/CD8 differentiation. PHA, but not anti$\mathrm{CD} 3 / \mathrm{CD} 28$, significantly increased the $\mathrm{CD} 8^{+}$population while simultaneously decreasing the $\mathrm{CD} 4^{+}$ population resulting in a significant $(p<0.0001)$ decrease of CD4:CD8 ratio relative to resting PBMC (1.7 to 0.9$)$. mAb activation also decreased the ratio but not as dramatically as PHA. Alloactivation, in comparison to the highly potent pan $\mathrm{T}$ cell activation, induced a more moderate proliferation of $\mathrm{CD}^{+}$ cells relative to resting PBMC.[3, 4, 25] The reduction in proliferation arose consequent to $<10 \%$ of $\mathrm{T}$ cells within a population typically being capable of allorecognition (Fig. 2B).[38, 39] Alloactivation similarly decreased $(p<0.01)$ the CD4:CD8 ratio.

The secretome products showed significant variability (at Day 10) in their effects when used to activate resting PBMC. As expected, resting PBMC treated with the SYN secretome were virtually identical to the resting PBMC with regards to both proliferation, subset analysis and CD4:CD8 ratio. Similarly, the tolerogenic TA1 preparation showed minimal proliferation and no substantive changes in the subset differentiation or the CD4:CD8 ratio. Of note, the small increase in $\mathrm{CD}^{+}$proliferation $(2.3 \pm 0.1 \%)$ supports earlier observations showing increased proliferation/differentiation of regulatory $T$ cells (Treg; $\mathrm{CD}^{+}{ }^{+}$oxp $^{+}$) in TA1-treated PBMC.[21] However, in contrast to the SYN and TA1 products, IA1 and IA2 showed significant variation from both the resting PBMC and from each other (Fig. 2C). IA1, derived from a control MLR, significantly increased the total $\mathrm{CD} 3^{+}$cell proliferation and decreased the relative abundance of $\mathrm{CD} 4^{+}$cells (38.8 $\pm 3.4 \%$ versus $56.0 \pm 1.5 \%$ for resting PBMC). Consequent to this change, the IA1 CD 4:CD8 ratio was significantly reduced $(1.0 \pm 0.1 ; p<0.001)$ suggestive of a pro-inflammatory state and similar to that noted with the pan T cell activators. Interestingly, the cancer cell (HeLa) stimulated biologic IA2 while inducing a similar level of $\mathrm{CD}^{+}$cell proliferation, showed dramatically different phenotype distribution (Fig. $2 \mathrm{C}$ ). In contrast to IA1, which reduced the CD4:CD8 ratio, IA2 significantly increased the ratio relative to both IA1 and the resting PBMC $(2.1 \pm 0.4,1.0 \pm 0.1$, and $1.7 \pm$ 
0.1 ; respectively) consequent to a decrease in $\mathrm{CD} 8^{+}$cells. These findings further support our previous study suggesting that the anti-HeLa effects of IA2 treated PBMC were distinct from that of IA1 treated PBMC.[4, 25]

Interestingly, both IA1 and IA2 activation also resulted in a significant expansion $(p<0.05)$ of double negative ( $\left.\mathrm{CD} 4^{-} \mathrm{CD} 8^{-}\right) \mathrm{T}$ cells. These double negative cells, while poorly understood, have been implicated in both inflammation and as regulatory cells. [40] Under certain activating stimuli, $\mathrm{CD}^{-}{ }^{-} \mathrm{CD}^{-}{ }^{-} \mathrm{T}$ cells can display the phenotype of effector cells that are capable of producing pro-inflammatory cytokines e.g., IFNY, IL-17A, and the phenotype of suppressor cells that secrete immune regulatory cytokine (e.g., IL-10). Consequently, $\mathrm{CD} 4^{-} \mathrm{CD} 8^{-} \mathrm{T}$ cells have been described in promoting neuroinflammation after ischemic stroke, [41] as well as preventing autoimmune diseases and graft-versus-host disease (GvHD).[42-45] Interestingly, a recent study revealed the potential role of $\mathrm{CD}^{-}{ }^{-} \mathrm{CD} 8^{-} \mathrm{T}$ cells in limiting alloreactive immune response by suppressing the CD4 T cell functionality.[46] Surprisingly perhaps, TA1 treatment reduced the $\mathrm{CD} 4^{-} \mathrm{CD} 8^{-}$population despite previous research documenting its potent tolerogenic activity in a murine model of Type 1 diabetes.[3]

In contrast, double positive $\left(\mathrm{CD} 4^{+} \mathrm{CD} 8^{+}\right) \mathrm{T}$ cells were reduced in the TA1, IA1 and IA2 $(0.7 \pm 0.0,3.0 \pm 0.5$, and $1.2 \pm 0.1 \%$, respectively) activated cells relative to Pan T cell activation (PHA: $7.0 \pm 2.3 \%$; and AntiCD3: $5.7 \pm 0.5 \%)$, and alloactivation $(6.0 \pm 0.7 \%)$. The biologic functions of $\mathrm{CD} 4^{+} \mathrm{CD} 8^{+} \mathrm{T}$ cells remain unclear with, perhaps most, studies reporting a pro-inflammatory role in cancers though some evidence of an immunosuppressive role have been reported.[47-50] $\mathrm{CD} 4^{+} \mathrm{CD} 8^{+} \mathrm{T}$ cells are increased in urologic cancers patients and found to be high type-2 cytokine producers favoring a Th2 response in vitro while inhibiting Th1 cells known to play a crucial role in anti-tumor immunity.[50] Hence, the decrease in the $\mathrm{CD} 4^{+} \mathrm{CD} 8^{+}$cells post secretome (TA1, IA1 and IA2) activation, relative to Pan T cell or allo- activation, may have clinical benefit and be indicative of the secretomes more 'restrained' immunoactivation.

To determine how these differential T cell activation strategies (i.e., Pan T cell, allorecognition and secretome) affected resting human PBMC, the intracellular expression of 84 miRNA involved in immunopathology pathways were previously assessed.[3, 4, 25] To further investigate the differential effects of the three T cell activation strategies on resting PBMC, the 'relative pattern of miRNA expression' was examined using subset of thirteen differentially expressed miRNA (Fig. 3A; miRNA were selected via clustergram heatmap[4] and/or $\log _{2}$ fold change analysis). The putative/described functions for these miRNA are summarized in Supplementary Table 1S. $[3,4,25]$ However, it is important to note that the 'putative' functions of the distinct miRNA can vary significantly depending on the biological (e.g., prostate versus T cell) model used. Moreover, consequent to the low genetic fidelity of a single miRNA, we propose that the most informative approach is the analysis of the differential activation strategies on the relative 'pattern of miRNA expression' across a number of miRNA (Fig. 3).

As shown, the Pan T cell activators PHA and anti-CD3/CD28 demonstrated significant similarities in their 'patterns of miRNA expression' and $\mathrm{CD}^{+}$proliferation (Fig. 3B). While these Pan T cell activators are 
commonly used as activation surrogates for allorecognition and/or to enhance cytokine expression levels prior to flow analysis (anti-CD3/CD28), comparison of the miRNA expression profiles show significant variances from the allorecognition expression profile (Control MLR; Fig. 3B). Surprisingly, minimal differences within the group of 13 miRNA were noted between the control- and mPEG- MLRs despite significant differences in T cell proliferation. However, in the context of the overall 84 miRNA screened, distinct patterns are quite apparent (Supplemental Table 2S and Scott et al.[25]). In contrast to both Pan T cell activators and allorecognition, secretome (IA1, IA2 and TA1) activation gave rise to dramatically reduced levels of proliferation and subtler changes, though highly distinct, changes in miRNA expression (Fig. 3). The IA1 secretome (Proliferation of $12.2 \pm 1.2 \%$ ) yielded a distinct miRNA expression profile from those of the Pan-T cell activators; though a muted similarity in the peaks and troughs is discernable. In contrast, the tolerogenic TA1 secretome induced minimal proliferation $(2.3 \pm 0.1 \%)$ and produced a miRNA pattern that varied significantly from the pro-inflammatory and proliferation inducing Pan $T$ cell and IA1 activators (Fig. 3). Indeed, previous clustergram heatmap analysis showed that miRNA expression induced by TA1 resembled resting and SYN treated PBMC but induced a potent Treg-mediated tolerogenic effect both in vitro and in vivo.[3, 4, 21, 25] Interestingly, the HeLa-PBMC manufactured IA2, while exerting a proliferative effect $(10.7 \pm 0.5 \%)$ similar to IA1, varied significantly from IA1 miRNA pattern (Fig. 3). Importantly, the differential patterns of expression (Fig. 3B) of the IA1, IA2 and TA1 miRNA were associated with differential biological effects on the naive PBMC with TA1 inducing systemic tolerance (in vitro and in vivo) and IA1 enhancing PBMC-mediated inhibition of cancer cell growth while IA2 exhibiting direct toxicity (apoptosis) of cancer cells. [3, 4] Importantly, the distinct expression patterns of miRNA between the Pan T cell activators (PHA and anti-CD3/CD28), alloactivation the secretome products induced activation translated into dramatically different biological responses.[3, $4,25]$

Consequent to our interests in using the IA1 secretome to activate resting PBMC to more efficiently cancer cells while preventing adverse events (e.g., cytokine storms), we more directly compared the miRNAs expression profile of IA1 to pan-T cell (anti-CD3/CD28; Fig. 4A), TA1 (Fig. 4B) and IA2 (Fig. 4C) activation via volcano plot analyses. Volcano plot analyses visualizes the differential miRNA data based on log scale changes and allows for statistical comparison of the expression of discreet miRNA between two samples (e.g., IA1 versus IA2) - but largely misses out on the overall PATTERN of changes seen with clustergram heatmaps[4] and Log2 fold change. As noted in Fig. 4A, distinct differences are noted between IA1 and anti-CD3/CD28. IA1 significantly $(p<0.05)$ upregulated the expression of miR-125b-5p and miR-451a relative to anti-CD3/CD28, while miR-18a-5p, miR-17-5p, miR-20a-5p and miR-135b-5p were downregulated. Similar to Fig. 3 multiple other miRNA were also differentially expressed between IA1 and anti-CD3/CD28 activation though they did not reach significance in the volcano plot analyses (though if compared to resting PBMC they are different). Interestingly, the miRNA expression profiles between IA1 and TA1 were not statistically significantly different (Fig. 4B), though, as also seen in Fig. 3, miR-298, miR-214-3p, miR-302a-3p and miR-206 were over-expressed in IA1 relative to TA1. Finally, the expression of miR-149-5p and miR-18b-5p were significantly $(p<0.05)$ upregulated in PBMC treated with IA1 when compared to the same donor PBMC treated with IA2 (Fig. 4C). 
In sum, previous clustergram,[4] and our current studies utilizing $\log _{2}$ fold change and volcano plot analyses demonstrate the differential activation strategies yielded dramatically different miRNA expression profiles that in turn resulted in significant differences in T cell activation and subset differentiation. In order to better understand these differences, an integrative Venn diagram analysis was done using all three sets of data (Fig. 5) in order to differentially compare the pan T cell, allorecognition and secretome activation. As demonstrated, Pan T cell activation using PHA and anti-CD3/CD28 yielded similar, though not identical, changes in miRNA expression (solid circles = over expression; dashed circles $=$ reduced expression; overlap are miRNA in common). For further comparison purposes, we averaged the miRNA expression profile and proliferation rates of PHA and anti-CD3/CD28 to represent the efficacy of pan T cell activation strategy. In contrast to Pan T cell activation, the miRNA changes induced by allorecognition were much more discreet (relative to resting PBMC) and highly limited when compared to the Pan T cell activators. Moreover, allorecognition resulted in a significant reduction in cell proliferation (Pan T: $86.3 \%$ versus $30.9 \%$ for Allorecognition). Similar to the allorecognition response, the allo-derived IA1 secretome also reduced the miRNA response pattern relative to Pan T cell activation and, not surprisingly, was similar to the pattern of expression observed in the alloresponse but with the increased expression of miR-298 and decreased expression of miR-206 and miR-214-3p. While some miRNA are in common to the Pan T cell, Allo, IA1 and IA2 pro-inflammatory responses (overlaps in Venn diagrams), some of these (e.g., miR-155-5p) are also implicated in the tolerogenic TA1 and mPEG-Allo responses. This again argues that the 'pattern of miRNA expression'(Fig. 3B) encompassing increases, decreases and static levels of multiple, rather than a specific (single or small number), of miRNA is crucial.

Importantly, the biomanufaturing process is of importance. This is most obvious in comparing the MLR vs mPEG-MLR and IA1 vs IA2 miRNA patterns. While IA1 and IA2 stimulate similar proliferative effects (12.2 \pm 1.2 and $10.7 \pm 0.5 \%$, respectively), their impacts on CD4/CD8 differentiation (Fig. 2) were vastly different and, as observed in our previous study, IA1 and IA2 exhibited distinct biological activities and anti-cancer mechanisms.[3, 4, 25] Indeed, as shown in Fig. 5, PBMC pre-treated with IA1 and IA2 induced entirely different miRNA expression that resulted in vastly different responses to cancer cells. IA2 but not $I A 1$, increased the expression of cellular miR-29b-3p which has been shown to promote cellular apoptosis in cancers. [51, 52] Consistently, miR-181a-5p, an oncogene in various tumors suppressing apoptosis, was downregulated by IA2, further supporting our previous findings that IA2 inhibited cancer cell proliferation via an apoptosis-associated mechanism.[53-55]

In conclusion, these studies demonstrate that pan T cell activators, alloactivation and secretome-based therapeutics induced differential patterns of miRNA expression in leukocytes, which governs/reflects significant differences in cell proliferation, differentiation and immunological activity. Pan $\mathrm{T}$ cell activators induced massive miRNA alteration profiles and T cell proliferation relative to resting cells. AlloMLR demonstrated a more discriminatory alteration of miRNA expression relative to pan T cell activators, while mPEG-MLR diminished allorecognition related miRNA expression. Importantly, IA1 and TA1 secretome derived from allo- and mPEG-MLR respectively, exerted similar miRNA pattern of change and immunomodulatory efficacies to its origin MLR response. In contrast, resting cell generated secretome 
SYN had minimal effects on recipient resting PBMC. Of interest, the HeLa-MLR derived IA2 therapeutic exhibited distinct alterations to the leukocyte miRNA expression profile, suggesting an apoptosisassociated immunomodulatory and anti-cancer pathway.

\section{Discussion}

Systemic, and in some cases localized, immunomodulation of the immune response is proving to be a potent weapon in the treatment of both autoimmune diseases and cancers. While multiple drugs (e.g., cyclosporine, PHA) and biologics (e.g., etanercept and anti-CD3) have been explored and, in some case used successfully in the clinic, these agents do not fundamentally reset the immune response. Research from our laboratory has demonstrated that polymer-based bioengineering of immune cells can be used either directly or indirectly, via secretome miRNA, to systemically modify the immune response.[3, 4, 19$24,56-58]$ of potential therapeutic value, the bioreactor-based manufacturing of secretome therapeutics (i.e., TA1 and IA1) have been explored more in depth and compared to existing Pan T cell activators (PHA and anti-CD3/anti-CD28) as well as the MHC-dependent alloresponse.

In this study we demonstrated that controlled and reproducible immunomodulation (cell proliferation and $\mathrm{CD} 4^{+} / \mathrm{CD} 8^{+} \mathrm{T}$ subset differentiation; Fig. 2 ) could be accomplished using the TA1 and IA1 secretome biotherapeutics. Mechanistically, the miRNA-expression profiles in resting PBMC were analyzed in the Pan T cell, allorecognition and secretome-based activation (Figs. 3-5). In these studies, as well as our previous publications, PEGylated-PBMC as well as TA1, derived from an mPEG-MLR, was shown to induce a systemic and persistent tolerogenic state.[3, 19, 21-25] In contrast, the allorecognition-based IA1 induced a more controlled pro-inflammatory response when compared to either Pan T Cell activators or the alloresponse itself (Figs. 2, 5). The controlled inflammatory response induced by IA1 is important as stronger pro-inflammatory approaches (e.g., mitogen and even allorecognition) can generate overly robust responses leading to bystander cell injury and, in worst case scenarios, cytokine release syndrome. $[3,4,19,21-25]$ In aggregate, the results shown here and in our previous publications suggest that secretome-based therapeutics could be potent, but controllable, tools for immunomodulation.

Immune cell activation results in multiple changes within the cell itself and is ultimately defined by cell proliferation and differentiation. The ratio of CD4:CD8 cells can be indicative of $T$ cell priming and tumor immunity.[59, 60] IA1, similar to the control-MLR, anti-CD3/CD28 and PHA, significantly decreased the ratio of CD4:CD8 cells. In contrast, despite its similar overall (i.e., CD3) proliferation rate, IA2 increased the CD4:CD8 ratio (Fig. 2). These findings demonstrated that IA1, as well as the Pan T cell and Allo activators, induced a strengthened $\mathrm{CD} 8^{+} \mathrm{T}$ cell response while IA2 primarily induced a $\mathrm{CD} 4^{+}$response. More research is needed to determine the interplay of the miRNA expression profiles with $\mathrm{T}$ cell differentiation.

Importantly however, as previously reported, the secretome products resulting in biologically distinct immunomodulatory effects. IA1 pre-treatment of resting PBMC significantly enhanced cell-mediated killing of cancer cells while IA2 exerted less effect on PBMC but induced apoptosis of cancer cells, but not 
PBMC.[4] In contrast to IA1 and IA2, TA1 (as well as allogeneic mPEG-WBC) is a potent inducer of tolerance both in vitro and in vivo via the production of Treg cells and the prevention of allo/xenorecognition.[3, 4, 21, 23-25] Indeed, in a murine model of Type 1 Diabetes pretreatment of NOD mice at 7 weeks of age with a single course of TA1 dramatically inhibited the development of diabetes and resulted in improved platelet histology.[3]

While immunologists have focused on cytokine/chemokine immunotherapy, miRNA may prove to be biologically more useful in that, with appropriate formulations, the strength and type of the immune response may be more titratable. miRNA have been increasingly found to be key bioregulatory messengers for both externally (i.e., extracellular miRNA) effecting change as well as key intracellular effectors of gene activation and mRNA expression. Indeed, the importance of miRNA in immune cell proliferation and differentiation has been extensively studied.[31-33,61-68] Moreover, the utility of miRNA in cancer diagnosis (i.e., biomarkers) and treatment (small interfering RNA; siRNA) has gained increasing attention during the past decade.[29, 69-80] As documented in this study, Pan T cell, allorecognition and secretome activation of resting PBMC induce dramatically different miRNA expression patterns within the treated PBMC (Figs. 3-5) that lead to different proliferation and differentiation responses. Typically, due to the inherent reductionist nature of science, large increases or decreases of a single miRNA have been focused on as being responsible for the observed changes in proliferation and differentiation in both autoimmune diseases and in proinflammatory states. However, our understanding of miRNA-mediated bioregulation is still in its infancy. In light of the fact that a single miRNA can interact with hundreds of genes, and a single gene can interact with tens to hundreds of miRNA, this 'low fidelity' is suggestive that 'distinct patterns of miRNA expression' rather than changes in a single miRNA are key to inducing complex (e.g., tolerance or controlled inflammation) bioregulatory changes.[3, 4, 25, 62, 81] Indeed, examining the miRNA expression profiles (Figs. 3-5) induced by Pan T cell activators (PHA or anti-CD3-CD28), allorecognition, or our secretome-derived TA1 and IA1 products shows significant variations in both cell phenotypes and the miRNA expression profiles. Singling out a single miRNA is difficult to do. For example, miR-155-5p is a crucial component of both tolerogenic and pro-inflammatory responses, but other supporting miRNA differ (e.g., Fig. 5). However, if one appreciates the complexity of miRNA, one can begin to look at 'patterns of expression' as governing the bioregulatory response. Schematically this bioregulatory process is described in Fig. 6A in which the unique pattern of miRNA expression observed in Fig. 3B are presented and govern the biologic response in a 'lock and key' manner. Hence, the 'pattern of expression' of TA1 replicates the expression profile to induce a tolerogenic response (i.e., increased Treg, decreased Teff; $[3,4,21,25]$ Fig. 6B) while the IA1 expression profile cannot induce tolerance due to its 'pattern disparity'. In contrast, IA1 effectively unlocks a controlled inflammatory response (Fig. 6C) that is in stark contrast to the Pan T cell or even alloresponse induced inflammation which are characterized by significantly more expansive miRNA expression profiles (Figs. 4-5). Moreover, it is possible to also hypothesize that partial pattern homology could induce a partial response (Fig. 6D); indeed, while TA1 is shown as the example, IA1 is a clear subset of the Pan T cell and Alloresponse miRNA response patterns (Fig. 5). The complexity of this 'lock and key'regulatory mechanism readily explains the often highly disparate functions assigned to a single miRNA in the 
literature (see Supplementary Tables $1 \mathrm{~S}$ and $2 \mathrm{~S}$ ). Hence, despite the reductionist nature of science, understanding miRNA bioregulation may need to become more focused on the overall expression patterns including not just large changes (up/down), but more modest increases or decreases, and even miRNA that remain static responses during complex biological responses. By mimicking the 'pattern of miRNA expression'it may be possible to produce secretome, or purified miRNA, products that can replicate the desired biological response. Indeed, the TA1 therapeutic induced a systemic in vivo tolerance resulting in disease attenuation in the NOD mouse model of Type 1 diabetes.[3] In contrast, treatment of resting PBMC with the IA1 therapeutic significantly enhanced their anti-cancer efficacy in vitro.[4] Of note, secretome-derived miRNA-based therapeutics could be used either independently or in conjunction with other cell-based therapies (e.g., CAR T cells).

Finally, an important finding of this study is that Pan T cell activation bears little resemblance to allorecognition with regards to the miRNA induced, which, in turn, will govern the regulation and differentiation of resting T cells. While many publications have utilized pan T cell activators (e.g., PHA or anti-CD3/anti-CD28) to model T cell-mediated pathologies (e.g., allorecognition, transplant rejection, GvHD, autoimmunity and inflammation), $[12,82]$ our results clearly indicate that there are very significant differences in the miRNA response pattern of human PBMC at 72 hours (well into the proliferation and differentiation cycle) between the activation strategies (Fig. 5). Indeed, pan T cell stimulators ( 90\% activation) induce massive miRNA alterations (Figs. 2-5), that yield non-specific overactivation and significant bystander injuries.[82-84] In contrast, allorecognition generates a more discriminatory $T$ cell response (proliferation/differentiation and miRNA expression; Figs. 2-5) as only $1-10 \%$ of an individual's T lymphocytes are alloreactive.[38, 39] Despite the 'low' number of potentially reactive cells, the alloresponse is still quite potent as exemplified by the severity of GvHD.[85] Not surprisingly, the alloresponse has been studied in the context of cancer immunity for decades.[18, 39, 86-88] Although mitogens and anti-CD3/CD28 have been widely used to activate and expand immune cells ex vivo in cancer immunotherapies, our findings suggest that pan $T$ cell activators do not model normal allorecognition-based stimulation and a reliance of these agents may lead to artifactual conclusion regarding immune responses, and could underlie the cytokine release syndrome commonly seen in overly robust $T$ cell responses to 'non-self'.[82-84]

\section{Conclusions}

T cell activation is a crucial element of the immune response. Recent studies have shown that miRNAs play a key role in tuning the immune response toward either an inflammatory or tolerogenic pathway. However, as demonstrated here, activation stimuli show significant variations in their generation of miRNA and, hence, the induced immunological response (strength, $T$ cell differentiation, inflammation, tolerance, etc). Indeed, our findings highlight the significant disparity between mitogen and alloactivation induced proliferation suggesting that mitogen stimulation is a poor model of 'normal' $T$ cell activation and may underlie their adverse effects when used clinically. In the development of miRNA-based therapeutics, we propose that the 'pattern of miRNA production', rather than a single miRNA, is of paramount importance as a single miRNA is 'low fidelity' in that it can interact with 10-100's of genes. 
Thus, we propose that miRNA-based bioregulation occurs via a 'lock and key' mechanism based on the 'pattern of miRNA expression' of a number of miRNA including increased, decreased and unchanged miRNA. In light of this observation, the development of miRNA-based therapeutics may be best achieved, NOT by focusing on a single miRNA, but rather via a cocktail of miRNA that mimic (at least partially) the naturally occurring miRNA expression profiles leading to controlled inflammation (e.g., IA1) or tolerance induction (e.g., TA1). To this end, the biomanufacturing of immunomodulatory, miRNA-enriched, secretome biotherapeutics may provide potent tools for the systemic treatment of both autoimmune diseases (TA1) and cancer (IA1).[3, 4, 25] The successful development of secretome-derived, miRNA therapeutics that replicate specific bioregulatory events may prove useful in the treatment of autoimmune diseases or enhancing the endogenous immune response to cancer while reducing the potential adverse risks of more non-specific immunomodulatory approaches.

\section{Methods And Materials}

\section{Human PBMC}

All human experiments were done in accordance with the University of British Columbia Clinical Research Ethics Board and the Code of Ethics of the World Medical Association (Declaration of Helsinki). The PBMC were isolated from donor whole blood using Histopaque-1077 (Sigma-Aldrich, St. Louis, MO) as described before.[3, 4, 21, 24] Human PBMC were washed and resuspended in AIM V media (research grade; ThermoFisher Scientific, Grand Island, NY).

\section{Differential Effects of Activation Strategies on Resting Leukocytes}

The effects of pan T cell activators [i.e., anti-CD3/anti-CD28 and mitogen (phytohemagglutinin; PHA)], alloactivators (i.e., control MLR and camouflaged MLR) and secretomes (i.e., SYN, TA1, IA1 and IA2) on the activation of resting leukocytes were compared (Fig. 1). In pan T cell activation, freshly isolated human PBMC were stimulated with anti-human CD3e in the presence of soluble anti-human CD28 for 3 days, or with PHA for 4 days as previously described (Fig. 1A).[4] Alloactivation was conducted in an MLR system with or without succinimidyl valerate activated (SVA) MPEG (Laysan Bio Inc. Arab, AL) for 10 days (Fig. 1B).[4] Effects of alloactivation were compared to untreated resting PBMC. To explore the immunomodulatory effects of alloactivation-secretome-derived therapeutics, cell-free TA1 and IA1 biologics were produced from MPEG-MLR and MLR respectively. Cell secretions from untreated resting PBMC were collected as SYN.[4] A lymphocyte-cancer cell (HeLa) biotherapeutic IA2 was concurrently developed from a HeLa-MLR as previously described.[4] In allo- and secretome activation studies, proliferation and phenotyping of treated PBMC were measured at day 10 (Fig. 1C). For all activation strategies, PBMC miRNA expression profiles were measured at 72 hours post treatment.

\section{T Cell Proliferation and Phenotyping}

The $T$ cell lymphocyte subpopulations $\left(\mathrm{CD} 3^{+} \mathrm{CD} 4^{+}\right.$and $\left.\mathrm{CD} 3^{+} \mathrm{CD} 8^{+}\right)$were measured using fluorescently labeled anti-CD3, CD4 and CD8 monoclonal antibodies (mAb; BD Pharmingen, San Jose, CA). All samples 
were acquired using the FACSCalibur flow cytometer and CellQuest Pro software (BD Biosciences, San Jose, CA) for both acquisition and analysis.

\section{Leukocyte miRNA Expression}

Total RNA was extracted from resting PBMC \pm treatment (anti-CD3/anti-CD28, PHA, MLR \pm mPEG, SYN, $I A 1, I A 2$ and TA1,) following 72 hours incubation using the mirVana ${ }^{\text {TM }}$ PARIS ${ }^{\text {TM }}$ kit (Ambion, Life Technologies, Grand Island, NY). Following processing, the highly enriched small RNA fraction containing miRNA was prepared using RNase/DNase free water. To partially characterize and quantify the relative abundance the miRNA species present in the resting and differentially activated PBMC, quantitative reverse transcription polymerase chain reaction (qRT-PCR) was done using the miScript miRNA PCR Array system (Qiagen, Frederick, MD) for the human immunopathology pathway as described before.[3, 4] The data shown represent three biological replicates analyzed independently by qRT-PCR.

\section{Statistical Analysis}

All data were expressed as mean \pm standard error mean (SEM). A minimum of three independent experiments were performed in duplicates for all studies. Data analysis was conducted using GraphPad Prism 6.0 (GraphPad Software, Inc., San Diego, CA). For comparison of means, a one-way analysis of variance (ANOVA) was performed. When significant differences were found, a post-hoc Tukey test was conducted for pair-wise comparison of means. For significance, a minimum p value of $<0.05$ (labeled

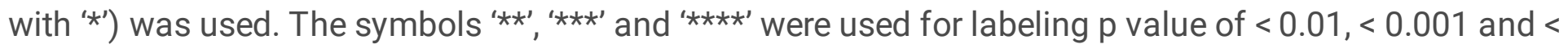
0.0001 , respectively.

\section{Abbreviations}

miRNA

microRNA; PBMC:Peripheral Blood Mononuclear Cells; $\mathrm{CD}^{+}$:Pan T lymphocyte marker; $\mathrm{CD}^{+}: \mathrm{CD}^{+}$subset (CD3 $\left.{ }^{+} \mathrm{CD} 4^{+} \mathrm{CD} 8\right)$; $\mathrm{CD}^{+}: \mathrm{CD}^{+}$subset $\left(\mathrm{CD} 3^{+} \mathrm{CD} 4^{-} \mathrm{CD} 8^{+}\right)$; cytotoxic $\mathrm{T}$ cells $(\mathrm{CTL})$; Treg:CD $3^{+} \mathrm{CD} 4^{+} \mathrm{Foxp}^{+} \mathrm{T}^{-}$ cell; promotes tolerance; Teff:CD $3^{+} \mathrm{CD} 4^{+} \mathrm{T}$ effector cell encompassing subsets such as Th17 and Th1; mPEG:methoxypolyethylene glycol; PHA:phytohemagglutinin; activates 90\% of T cells; Anti-CD3/antiCD28:monoclonal antibodies directed against CD3 and ; activates 80\% of T cells; Control-MLR:Mixed Lymphocyte Reaction (MLR); proliferation occurs due to allorecognition; mPEG-MLR:MLR reaction in which one donor population has been modified via the covalent grafting of mPEG; HeLa:Epithelial cancer cell line; SYN:control secretome product derived from resting PBMC; TA1:Tolerance Agent 1; derived from mPEG-MLR; IA1:Inflammatory Agent 1; derived from control-MLR; IA2:Inflammatory Agent 2; derived from PBMC-HeLa cell co-culture

\section{Declarations}

\section{Ethics approvals:}


All human experiments (i.e., blood donations) were reviewed and approved by the University of British Columbia Clinical Research Ethics Board (H02-70215) and done in accordance with the Code of Ethics of the World Medical Association (Declaration of Helsinki). All murine studies were done in accordance with the Canadian Council of Animal Care and approved by the University of British Columbia Animal Care Committee (A17-0220) and were conducted within the Centre for Disease Modeling at the University of British Columbia.

\section{Consent for publication}

All authors have read and approved the paper. If the paper is accepted, all authors will observe the terms of the license to publish.

\section{Availability of data and materials}

All data analyzed in this study are included within the included figures and tables, additional file 1, or are available from the authors upon reasonable request.

\section{Competing interests}

MDS and WMT are inventors on multiple issued and pending patents related to the disclosed technology. These patents are assigned to their primary employer: Canadian Blood Services (Ottawa, ON, Canada). Canadian Blood Services is a not-for-profit organization whose primary mission since its organization in 1998 is to collect, manufacture and manage blood and blood products for all of Canada (except Quebec). Canadian Blood Services is funded solely by the federal, provincial and territorial governments of Canada.

\section{Funding}

This work was supported by grants from the Canadian Institutes of Health Research (Grant No. 123317; MDS), Canadian Blood Services (MDS) and Health Canada (MDS). The views expressed herein do not necessarily represent the view of the federal government of Canada. The funders had no role in study design, data collection and analysis, decision to publish, or preparation of the manuscript.

\section{Authors' contributions}

MDS and $X Y$ were responsible for the design and analysis of the study. $X Y$ and WMT were responsible for the execution of the studies experiments. MDS, XY and WMT all participated in the writing of the manuscript. 


\section{Acknowledgments}

We thank the Canada Foundation for Innovation and the Michael Smith Foundation for Health Research for infrastructure funding at the University of British Columbia Centre for Blood Research. The authors would like to thank Ms. Luxi Wang for her laboratory assistance.

\section{References}

1. Naidoo J, Page DB, Wolchok JD. Immune modulation for cancer therapy. Br J Cancer. 2014;111:2214-9. 10.1038/bjc.2014.348.

2. Liebman HA. Immune modulation for autoimmune disorders: evolution of therapeutics. Semin Hematol. 2016;53(Suppl 1):23-6. 10.1053/j.seminhematol.2016.04.008.

3. Wang D, Shanina I, Toyofuku WM, Horwitz MS, Scott MD. Inhibition of Autoimmune Diabetes in NOD Mice by miRNA Therapy. PLoS One. 2015;10:e0145179. 10.1371/journal.pone.0145179.

4. Yang X, Kang N, Toyofuku WM, Scott MD. Enhancing the pro-inflammatory anti-cancer T cell response via biomanufactured, secretome-based, immunotherapeutics. Immunobiology. 2019;224:270-84. 10.1016/j.imbio.2018.12.003.

5. Fathman CG, Myers BD. Cyclosporine therapy for autoimmune disease. N Engl J Med. 1992;326:1693-5. 10.1056/NEJM199206183262509.

6. Feutren G. The optimal use of cyclosporin A in autoimmune diseases. J Autoimmun. 1992;5(Suppl A):183-95.

7. Scott LJ. Etanercept: a review of its use in autoimmune inflammatory diseases. Drugs. 2014;74:1379-410. 10.1007/s40265-014-0258-9.

8. Khanna M, Shirodkar MA, Gottlieb AB. Etanercept therapy in patients with autoimmunity and hepatitis C. J Dermatolog Treat. 2003;14:229-32. 10.1080/09546630310020470.

9. Mire-Sluis AR, Wickremasinghe RG, Hoffbrand AV, Timms AM, Francis GE. Human T lymphocytes stimulated by phytohaemagglutinin undergo a single round of cell division without a requirement for interleukin-2 or accessory cells. Immunology. 1987;60:7-12.

10. Deng C, Kaplan MJ, Yang J, Ray D, Zhang Z, McCune WJ, Hanash SM, Richardson BC. Decreased Ras-mitogen-activated protein kinase signaling may cause DNA hypomethylation in T lymphocytes from lupus patients. Arthritis Rheum. 2001;44:397-407. 10.1002/1529 - 0131(200102)44:2< 397::AID-ANR59 > 3.0.CO;2-N

11. Liao W, Lin JX, Leonard WJ. IL-2 family cytokines: new insights into the complex roles of IL-2 as a broad regulator of T helper cell differentiation. Curr Opin Immunol. 2011;23:598-604. 10.1016/j.coi.2011.08.003.

12. Trickett $A$, Kwan YL. T cell stimulation and expansion using anti-CD3/CD28 beads. J Immunol Methods. 2003;275:251-5. 10.1016/S0022-1759(03)00010 - 3.

13. Fabre JW. The allogeneic response and tumor immunity. Nat Med. 2001;7:649-52. 10.1038/89008. 
14. Starnes CO. Coley's toxins in perspective. Nature. 1992;357:11-2. 10.1038/357011a0.

15. Starnes CO. Coley's toxins. Nature. 1992;360:23. 10.1038/360023b0.

16. Barrett AJ. Mechanisms of the graft-versus-leukemia reaction. Stem Cells. 1997;15:248-58. 10.1002/stem.150248.

17. Barrett J, Childs R. The benefits of an alloresponse: graft-versus-tumor. J Hematother Stem Cell Res. 2000;9:347-54. 10.1089/15258160050079452.

18. Stathopoulos A, Samuelson C, Milbouw G, Hermanne JP, Schijns VE, Chen TC. Therapeutic vaccination against malignant gliomas based on allorecognition and syngeneic tumor antigens: proof of principle in two strains of rat. Vaccine. 2008;26:1764-72. 10.1016/j.vaccine.2008.01.039.

19. Murad KL, Gosselin EJ, Eaton JW, Scott MD. Stealth cells: prevention of major histocompatibility complex class II-mediated T-cell activation by cell surface modification. Blood. 1999;94:2135-41.

20. Le Y, Scott MD. Immunocamouflage: the biophysical basis of immunoprotection by grafted methoxypoly(ethylene glycol) (mPEG). Acta Biomater. 2010;6:2631-41.

10.1016/j.actbio.2010.01.031.

21. Kang N, Toyofuku WM, Yang X, Scott MD. Inhibition of allogeneic cytotoxic T cell (CD8(+)) proliferation via polymer-induced Treg (CD4(+)) cells. Acta Biomater. 2017;57:146-55. 10.1016/j.actbio.2017.04.025.

22. Kyluik-Price DL, Li L, Scott MD. Comparative efficacy of blood cell immunocamouflage by membrane grafting of methoxypoly(ethylene glycol) and polyethyloxazoline. Biomaterials. 2014;35:412-22. 10.1016/j.biomaterials.2013.09.016.

23. Kyluik-Price DL, Scott MD. Effects of methoxypoly (Ethylene glycol) mediated immunocamouflage on leukocyte surface marker detection, cell conjugation, activation and alloproliferation. Biomaterials. 2016;74:167-77. 10.1016/j.biomaterials.2015.09.047.

24. Wang D, Toyofuku WM, Chen AM, Scott MD. Induction of immunotolerance via mPEG grafting to allogeneic leukocytes. Biomaterials. 2011;32:9494-503. 10.1016/j.biomaterials.2011.08.061.

25. Scott MD, Wang D, Toyofuku WM, Yang X. Modulating the T Lymphocyte Immune Response via Secretome Produced miRNA: From Tolerance Induction to the Enhancement of the Anticancer Response. In: Fuchs O, editor. Cells of the Immune System. Croatia: IntechOpen; 2019.

26. Hammond SM. An overview of microRNAs. Adv Drug Deliv Rev. 2015;87:3-14. 10.1016/j.addr.2015.05.001.

27. Grigoryev YA, Kurian SM, Hart T, Nakorchevsky AA, Chen C, Campbell D, Head SR, Yates JR, Salomon DR. MicroRNA regulation of molecular networks mapped by global microRNA, mRNA, and protein expression in activated T lymphocytes. J Immunol. 2011;187:2233-43. 10.4049/jimmunol.1101233.

28. Neilson JR, Zheng GX, Burge CB, Sharp PA. Dynamic regulation of miRNA expression in ordered stages of cellular development. Genes Dev. 2007;21:578-89. 10.1101/gad.1522907.

29. Hayes J, Peruzzi PP, Lawler S. MicroRNAs in cancer: biomarkers, functions and therapy. Trends Mol Med. 2014;20:460-9. 10.1016/j.molmed.2014.06.005. 
30. Cortez MA, Calin GA. MicroRNA identification in plasma and serum: a new tool to diagnose and monitor diseases. Expert Opin Biol Ther. 2009;9:703-11. 10.1517/14712590902932889.

31. Teteloshvili N, Smigielska-Czepiel K, Kroesen BJ, Brouwer E, Kluiver J, Boots AM, van den Berg A. Tcell Activation Induces Dynamic Changes in miRNA Expression Patterns in CD4 and CD8 T-cell Subsets. Microrna. 2015;4:117-22. 10.2174/2211536604666150819194636.

32. Rossi RL, Rossetti G, Wenandy L, Curti S, Ripamonti A, Bonnal RJ, Birolo RS, Moro M, Crosti MC, Gruarin P, Maglie S, Marabita F, Mascheroni D, Parente V, Comelli M, Trabucchi E, De Francesco R, Geginat J, Abrignani S, Pagani M. Distinct microRNA signatures in human lymphocyte subsets and enforcement of the naive state in CD4 + T cells by the microRNA miR-125b. Nat Immunol. 2011;12:796-803. 10.1038/ni.2057.

33. Dudda JC, Salaun B, Ji Y, Palmer DC, Monnot GC, Merck E, Boudousquie C, Utzschneider DT, Escobar TM, Perret R, Muljo SA, Hebeisen M, Rufer N, Zehn D, Donda A, Restifo NP, Held W, Gattinoni L, Romero P. MicroRNA-155 is required for effector CD8 + T cell responses to virus infection and cancer. Immunity. 2013;38:742-53. 10.1016/j.immuni.2012.12.006.

34. Bartel DP. MicroRNAs: target recognition and regulatory functions. Cell. 2009;136:215-33. 10.1016/j.cell.2009.01.002.

35. Bartel DP. Metazoan MicroRNAs. Cell. 2018;173:20-51. 10.1016/j.cell.2018.03.006.

36. Shukla GC, Singh J, Barik S. MicroRNAs. Processing, Maturation, Target Recognition and Regulatory Functions. Mol Cell Pharmacol. 2011;3:83-92.

37. Grimson A, Farh KK, Johnston WK, Garrett-Engele P, Lim LP, Bartel DP. MicroRNA targeting specificity in mammals: determinants beyond seed pairing. Mol Cell. 2007;27:91-105.

10.1016/j.molcel.2007.06.017.

38. Nisbet NW, Simonsen M, Zaleski M. The frequency of antigen-sensitive cells in tissue transplantation. A commentary on clonal selection. J Exp Med. 1969;129:459-67. 10.1084/jem.129.3.459.

39. Basic Immunology E-Book Abul KA, Andrew HHL, Shiv P. Basic Immunology E-Book. 2015.

40. D'Acquisto F, Crompton T. CD3 + CD4-CD8- (double negative) T cells: saviours or villains of the immune response. Biochem Pharmacol. 2011;82:333-40. 10.1016/j.bcp.2011.05.019.

41. Meng H, Zhao H, Cao X, Hao J, Zhang H, Liu Y, Zhu MS, Fan L, Weng L, Qian L, Wang X, Xu Y. Doublenegative T cells remarkably promote neuroinflammation after ischemic stroke. Proc Natl Acad Sci U S A. 2019;116:5558-63. 10.1073/pnas.1814394116.

42. Ford MS, Chen W, Wong S, Li C, Vanama R, Elford AR, Asa SL, Ohashi PS, Zhang L. Peptide-activated double-negative T cells can prevent autoimmune type-1 diabetes development. Eur J Immunol. 2007;37:2234-41. 10.1002/eji.200636991.

43. Young KJ, DuTemple B, Phillips MJ, Zhang L. Inhibition of graft-versus-host disease by doublenegative regulatory T cells. J Immunol. 2003;171:134-41. 10.4049/jimmunol.171.1.134. 
44. Brandt $\mathrm{D}$, Hedrich $\mathrm{CM}$. TCRa $\beta^{+} \mathrm{CD} 3^{+} \mathrm{CD} 4^{-} \mathrm{CD} 8^{-}$(double negative) T cells in autoimmunity. Autoimmun Rev. 2018;17:422-30. 10.1016/j.autrev.2018.02.001.

45. Lev A, Simon AJ, Amariglio N, Rechavi G, Somech R. Thymic functions and gene expression profile distinct double-negative cells from single positive cells in the autoimmune lymphoproliferative syndrome. Autoimmun Rev. 2012;11:723-30. 10.1016/j.autrev.2012.01.002.

46. Haug T, Aigner M, Peuser MM, Strobl CD, Hildner K, Mougiakakos D, Bruns H, Mackensen A, Völkl S. Human Double-Negative Regulatory T-Cells Induce a Metabolic and Functional Switch in Effector TCells by Suppressing mTOR Activity. Front Immunol. 2019;10:883. 10.3389/fimmu.2019.00883.

47. Parel Y, Chizzolini C. CD4 + CD8 + double positive (DP) T cells in health and disease. Autoimmun Rev. 2004;3:215-20. 10.1016/j.autrev.2003.09.001.

48. Rahemtullah A, Reichard KK, Preffer FI, Harris NL, Hasserjian RP. A double-positive CD4 + CD8 + T-cell population is commonly found in nodular lymphocyte predominant Hodgkin lymphoma. Am J Clin Pathol. 2006;126:805-14. 10.1309/Y8KD-32QG-RYFN-1XQX.

49. Overgaard NH, Jung JW, Steptoe RJ, Wells JW. CD4+/CD8 + double-positive T cells: more than just a developmental stage. J Leukoc Biol. 2015;97:31-8. 10.1189/jlb.1RU0814-382.

50. Bohner P, Chevalier MF, Cesson V, Rodrigues-Dias SC, Dartiguenave F, Burruni R, Tawadros T, Valerio M, Lucca I, Nardelli-Haefliger D, Jichlinski P, Derré L. Double Positive CD4 + CD8 + T Cells Are Enriched in Urological Cancers and Favor T Helper-2 Polarization. Front Immunol. 2019;10:622. 10.3389/fimmu.2019.00622.

51. Li Y, Wang H, Tao K, Xiao Q, Huang Z, Zhong L, Cao W, Wen J, Feng W. miR-29b suppresses CML cell proliferation and induces apoptosis via regulation of BCR/ABL1 protein. Exp Cell Res. 2013;319:1094-101. 10.1016/j.yexcr.2013.02.002.

52. Zeng Y, Cui Z, Liu J, Chen J, Tang S. MicroRNA-29b-3p Promotes Human Retinal Microvascular Endothelial Cell Apoptosis via Blocking SIRT1 in Diabetic Retinopathy. Front Physiol. 2019;10:1621. 10.3389/fphys.2019.01621.

53. Yang M, Zhai X, Ge T, Yang C, Lou G. miR-181a-5p Promotes Proliferation and Invasion and Inhibits Apoptosis of Cervical Cancer Cells via Regulating Inositol Polyphosphate-5-Phosphatase A (INPP5A). Oncol Res. 2018;26:703-12. 10.3727/096504017 × 14982569377511.

54. Lai Y, Zhao L, Hu J, Quan J, Chen P, Xu J, Guan X, Lai Y, Ni L. microRNA-181a-5p functions as an oncogene in renal cell carcinoma. Mol Med Rep. 2018;17:8510-7. 10.3892/mmr.2018.8899.

55. Lai Y, Zhao L, Hu J, Quan J, Chen P, Xu J, Guan X, Lai Y, Ni L. [Corrigendum] microRNA-181a-5p functions as an oncogene in renal cell carcinoma. Mol Med Rep. 2018;18:1893. 10.3892/mmr.2018.9117.

56. Scott MD, Murad KL, Koumpouras F, Talbot M, Eaton JW. Chemical camouflage of antigenic determinants: stealth erythrocytes. Proc Natl Acad Sci U S A. 1997;94:7566-71. 10.1073/pnas.94.14.7566.

57. Le Y, Li L, Wang D, Scott MD. Immunocamouflage of latex surfaces by grafted methoxypoly(ethylene glycol) (mPEG): proteomic analysis of plasma protein adsorption. Sci China Life Sci. 2012;55:191- 
201. 10.1007/s11427-012-4290-2.

58. Wang D, Toyofuku WM, Scott MD. The potential utility of methoxypoly(ethylene glycol)-mediated prevention of rhesus blood group antigen $\mathrm{RhD}$ recognition in transfusion medicine. Biomaterials. 2012;33:3002-12. 10.1016/j.biomaterials.2011.12.041.

59. Miceli MC, Parnes JR. The roles of CD4 and CD8 in T cell activation. Semin Immunol. 1991;3:13341.

60. Shah W, Yan X, Jing L, Zhou Y, Chen H, Wang Y. A reversed CD4/CD8 ratio of tumor-infiltrating lymphocytes and a high percentage of CD4(+)FOXP3(+) regulatory $\mathrm{T}$ cells are significantly associated with clinical outcome in squamous cell carcinoma of the cervix. Cell Mol Immunol. 2011;8:59-66. 10.1038/cmi.2010.56.

61. Carissimi C, Carucci N, Colombo T, Piconese S, Azzalin G, Cipolletta E, Citarella F, Barnaba V, Macino G, Fulci V. miR-21 is a negative modulator of T-cell activation. Biochimie. 2014;107(Pt B):319-26. 10.1016/j.biochi.2014.09.021.

62. Chen CZ, Schaffert S, Fragoso R, Loh C. Regulation of immune responses and tolerance: the microRNA perspective. Immunol Rev. 2013;253:112-28. 10.1111/imr.12060.

63. Li QJ, Chau J, Ebert PJ, Sylvester G, Min H, Liu G, Braich R, Manoharan M, Soutschek J, Skare P, Klein LO, Davis MM, Chen CZ. miR-181a is an intrinsic modulator of T cell sensitivity and selection. Cell. 2007;129:147-61. 10.1016/j.cell.2007.03.008.

64. Lu LF, Thai TH, Calado DP, Chaudhry A, Kubo M, Tanaka K, Loeb GB, Lee H, Yoshimura A, Rajewsky K, Rudensky AY. Foxp3-dependent microRNA155 confers competitive fitness to regulatory $T$ cells by targeting SOCS1 protein. Immunity. 2009;30:80-91. 10.1016/j.immuni.2008.11.010.

65. Rusca N, Dehò L, Montagner S, Zielinski CE, Sica A, Sallusto F, Monticelli S. MiR-146a and NF-kB1 regulate mast cell survival and T lymphocyte differentiation. Mol Cell Biol. 2012;32:4432-44. 10.1128/MCB.00824 - 12.

66. Shin J, Xie D, Zhong XP. MicroRNA-34a enhances T cell activation by targeting diacylglycerol kinase

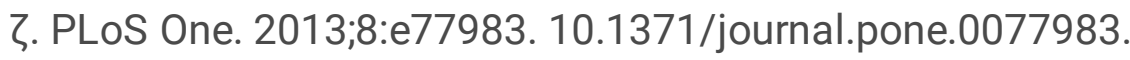

67. Wu H, Neilson JR, Kumar P, Manocha M, Shankar P, Sharp PA, Manjunath N. miRNA profiling of naïve, effector and memory CD8 T cells. PLoS One. 2007;2:e1020. 10.1371/journal.pone.0001020.

68. Xiao C, Calado DP, Galler G, Thai TH, Patterson HC, Wang J, Rajewsky N, Bender TP, Rajewsky K. MiR150 controls B cell differentiation by targeting the transcription factor c-Myb. Cell. 2007;131:146-59. 10.1016/j.cell.2007.07.021.

69. Chen X, Ba Y, Ma L, Cai X, Yin Y, Wang K, Guo J, Zhang Y, Chen J, Guo X, Li Q, Li X, Wang W, Zhang Y, Wang J, Jiang X, Xiang Y, Xu C, Zheng P, Zhang J, Li R, Zhang H, Shang X, Gong T, Ning G, Wang J, Zen K, Zhang J, Zhang CY. Characterization of microRNAs in serum: a novel class of biomarkers for diagnosis of cancer and other diseases. Cell Res. 2008;18:997-1006. 10.1038/cr.2008.282.

70. Chim SS, Shing TK, Hung EC, Leung TY, Lau TK, Chiu RW, Lo YM. Detection and characterization of placental microRNAs in maternal plasma. Clin Chem. 2008;54:482-90.

10.1373/clinchem.2007.097972. 
71. Kosaka N, Izumi H, Sekine K, Ochiya T. microRNA as a new immune-regulatory agent in breast milk. Silence. 2010;1:7. 10.1186/1758-907X-1-7.

72. Lawrie CH, Gal S, Dunlop HM, Pushkaran B, Liggins AP, Pulford K, Banham AH, Pezzella F, Boultwood $J$, Wainscoat JS, Hatton CS, Harris AL. Detection of elevated levels of tumour-associated microRNAs in serum of patients with diffuse large B-cell lymphoma. Br J Haematol. 2008;141:672-5. 10.1111/j.1365-2141.2008.07077.x.

73. Mitchell PS, Parkin RK, Kroh EM, Fritz BR, Wyman SK, Pogosova-Agadjanyan EL, Peterson A, Noteboom J, O'Briant KC, Allen A, Lin DW, Urban N, Drescher CW, Knudsen BS, Stirewalt DL, Gentleman R, Vessella RL, Nelson PS, Martin DB, Tewari M. Circulating microRNAs as stable bloodbased markers for cancer detection. Proc Natl Acad Sci U S A. 2008;105:10513-8. 10.1073/pnas.0804549105.

74. Park NJ, Zhou H, Elashoff D, Henson BS, Kastratovic DA, Abemayor E, Wong DT. Salivary microRNA: discovery, characterization, and clinical utility for oral cancer detection. Clin Cancer Res. 2009;15:5473-7. 10.1158/1078 - 0432.CCR-09-0736.

75. Weber JA, Baxter DH, Zhang S, Huang DY, Huang KH, Lee MJ, Galas DJ, Wang K. The microRNA spectrum in 12 body fluids. Clin Chem. 2010;56:1733-41. 10.1373/clinchem.2010.147405.

76. Chen $X$, Liang $H$, Zhang J, Zen $K$, Zhang CY. Secreted microRNAs: a new form of intercellular communication. Trends Cell Biol. 2012;22:125-32. 10.1016/j.tcb.2011.12.001.

77. Turchinovich A, Weiz L, Burwinkel B. Extracellular miRNAs: the mystery of their origin and function. Trends Biochem Sci. 2012;37:460-5. 10.1016/j.tibs.2012.08.003.

78. Bovy N, Blomme B, Frères P, Dederen S, Nivelles O, Lion M, Carnet O, Martial JA, Noël A, Thiry M, Jérusalem G, Josse C, Bours V, Tabruyn SP, Struman I. Endothelial exosomes contribute to the antitumor response during breast cancer neoadjuvant chemotherapy via microRNA transfer. Oncotarget. 2015;6:10253-66. 10.18632/oncotarget.3520.

79. Munich S, Sobo-Vujanovic A, Buchser WJ, Beer-Stolz D, Vujanovic NL. Dendritic cell exosomes directly kill tumor cells and activate natural killer cells via TNF superfamily ligands. Oncoimmunology. 2012;1:1074-83. 10.4161/onci.20897.

80. Okoye IS, Coomes SM, Pelly VS, Czieso S, Papayannopoulos V, Tolmachova T, Seabra MC, Wilson MS. MicroRNA-containing T-regulatory-cell-derived exosomes suppress pathogenic $T$ helper 1 cells. Immunity. 2014;41:89-103. 10.1016/j.immuni.2014.08.008.

81. Mavrakis KJ, Van Der Meulen J, Wolfe AL, Liu X, Mets E, Taghon T, Khan AA, Setty M, Setti M, Rondou $P$, Vandenberghe P, Delabesse E, Benoit Y, Socci NB, Leslie CS, Van Vlierberghe P, Speleman F, Wendel HG. A cooperative microRNA-tumor suppressor gene network in acute T-cell lymphoblastic leukemia (T-ALL). Nat Genet. 2011;43:673-8. 10.1038/ng.858.

82. Maciel RM, Miki SS, Nicolau W, Mendes NF. Peripheral blood T and B lymphocytes, in vitro stimulation with phytohemagglutinin, and sensitization with 2,4-dinitrochlorobenzene in Grave's disease. J Clin Endocrinol Metab. 1976;42:583-7. 10.1210/jcem-42-3-583. 
83. Han T, Takita H. Immunologic impairment in bronchogenic carcinoma: a study of lymphocyte response to phytohemagglutinin. Cancer. 1972;30:616-20.

84. Suntharalingam G, Perry MR, Ward S, Brett SJ, Castello-Cortes A, Brunner MD, Panoskaltsis N. Cytokine storm in a phase 1 trial of the anti-CD28 monoclonal antibody TGN1412. N Engl J Med. 2006;355:1018-28. 10.1056/NEJMoa063842.

85. Nowak J. Role of HLA in hematopoietic SCT. Bone Marrow Transplant. 2008;42(Suppl 2):71-6. $10.1038 / \mathrm{bmt} .2008 .288$.

86. de Gruijl TD, van den Eertwegh AJ, Pinedo HM, Scheper RJ. Whole-cell cancer vaccination: from autologous to allogeneic tumor- and dendritic cell-based vaccines. Cancer Immunol Immunother. 2008;57:1569-77. 10.1007/s00262-008-0536-z.

87. Gong J, Nikrui N, Chen D, Koido S, Wu Z, Tanaka Y, Cannistra S, Avigan D, Kufe D. Fusions of human ovarian carcinoma cells with autologous or allogeneic dendritic cells induce antitumor immunity. $\mathrm{J}$ Immunol. 2000;165:1705-11. 10.4049/jimmunol.165.3.1705.

88. Kugler A, Stuhler G, Walden P, Zöller G, Zobywalski A, Brossart P, Trefzer U, Ullrich S, Müller CA, Becker V, Gross AJ, Hemmerlein B, Kanz L, Müller GA, Ringert RH. Regression of human metastatic renal cell carcinoma after vaccination with tumor cell-dendritic cell hybrids. Nat Med. 2000;6:332-6. $10.1038 / 73193$.

\section{Figures}




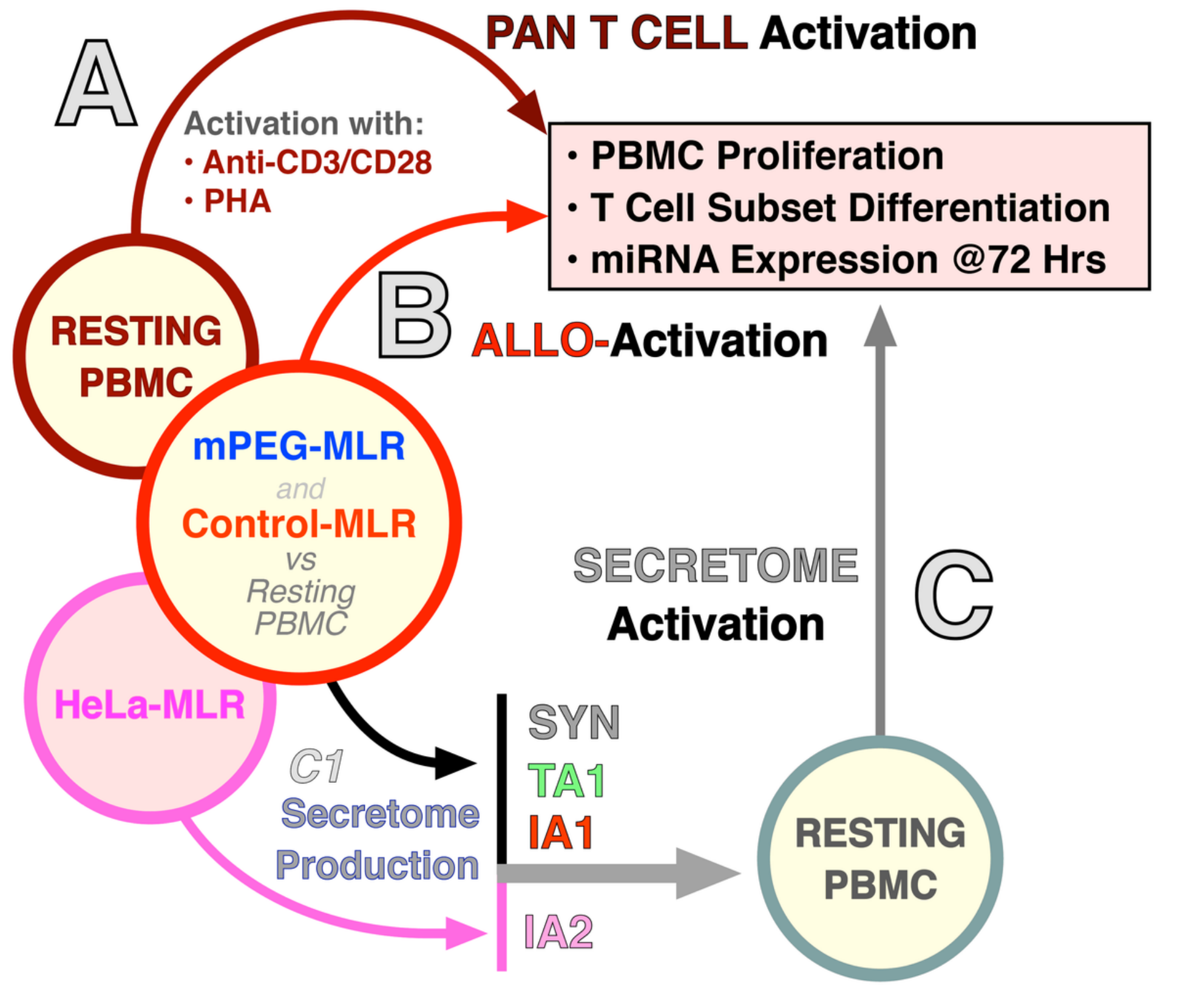

Figure 1

Schematic presentation of the three differential activation strategies (A-C: Pan T; Allorecognition; and Secretome) of PBMC activation studied. T cell proliferation and subset differentiation were measured via flow cytometry and leukocyte miRNA expression levels which were compared to untreated resting PBMC. Pan T cell activation (A) of freshly isolated human resting PBMC utilized anti-CD3/anti-CD28 or PHA. Alloactivation (B) was conducted via an MLR with or without MPEG (20 kDa mPEG; grafting concentration of $2 \mathrm{mM}$ ) immunocamouflage of one MHC-disparate donor. The negative control consisted of the untreated resting PBMC. Secretome activation consisted of two phases: production of the secretome therapeutics (C1) and the activation (C) of freshly collected donor PBMC with the manufactured secretome therapeutic. The SYN, TA1 and IA1 was derived from resting PBMC, mPEG-MLR and control MLR respectively. The IA2 secretome product was produced using an PBMC-cancer cell (HeLa) MLR model (HeLa-MLR). Proliferation and subset analysis of the different activation strategies 
were optimized as previously described.[4] miRNA profiles of the activated PBMC of the differential strategies (A-C) was done at 72 hours for and compared to untreated resting PBMC.

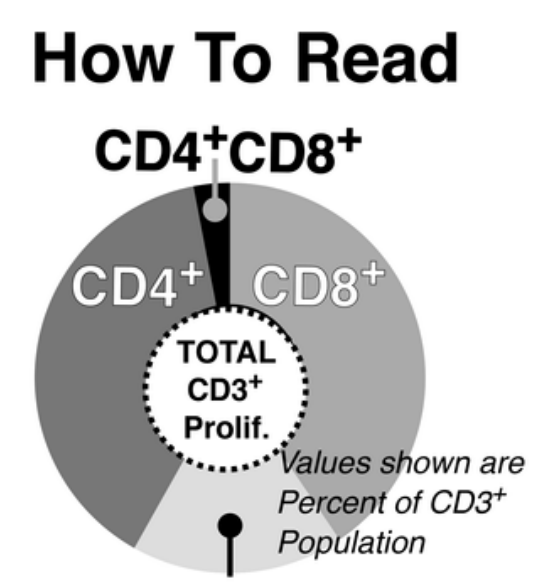

CD4"CD8-

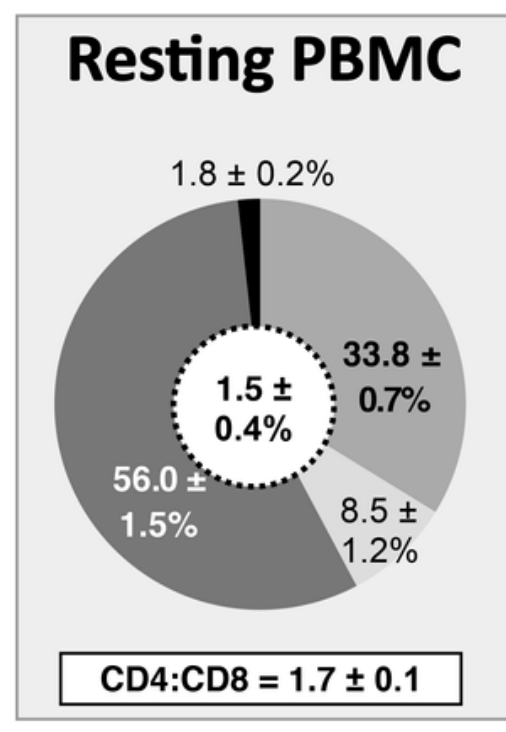

\section{Pan T Cell Activators}

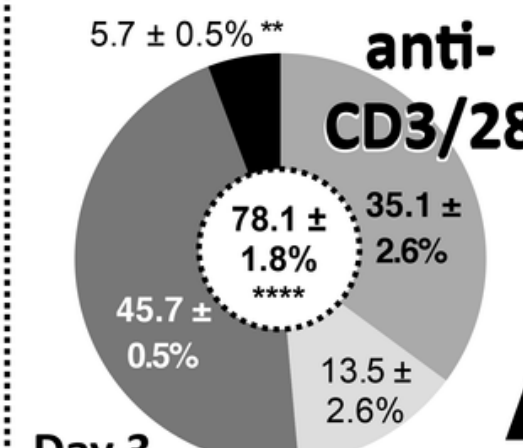

Day 3
CD4:CD8 Ratio

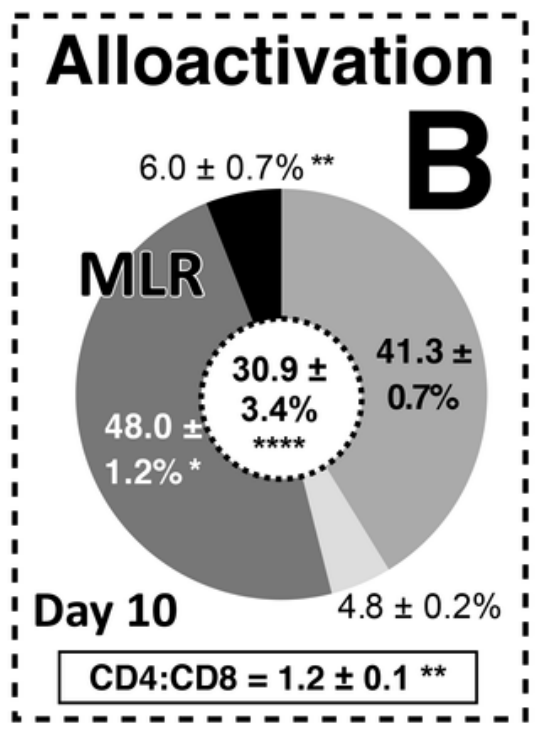

\section{Secretomes}
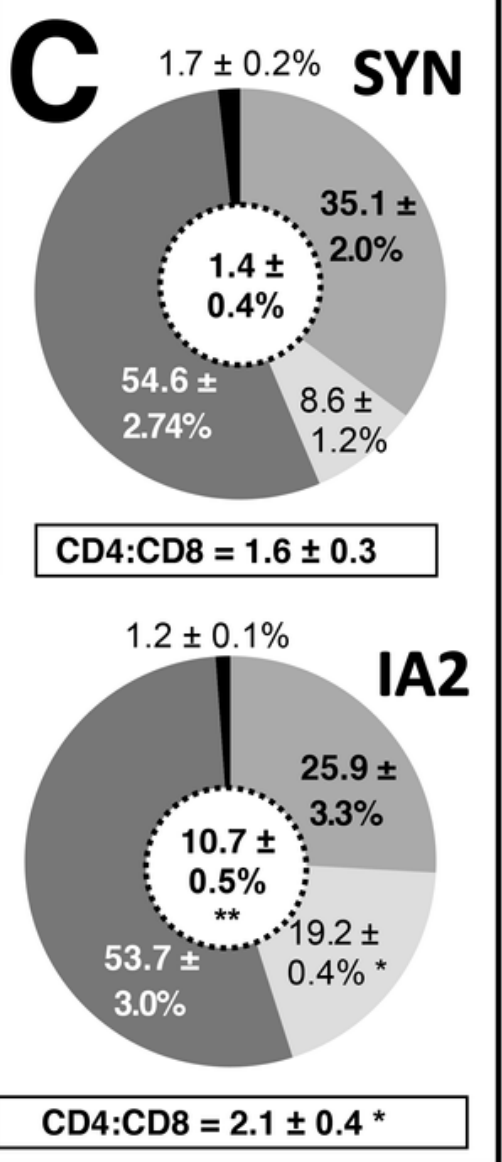

Figure 2

Effects of the different activation strategies on T cell CD4 \pm and CD8 \pm subset differentiation. Upper left panel describes the data presentation. Briefly the centre circle indicates total CD3+ proliferation[4] and the slices of the pie-chart denote percentages of the indicated CD4/CD8 subsets of the total CD3+ cells at the 
indicated day. Resting CD3+ PBMC are shown for comparison at 10 days incubation. Panel A: Results of the Pan T cell activators Anti-CD3/CD28 at Day 3 and PHA at Day 4. Panel B: Control MLR alloactivation at Day 10. Panel C: Secretome therapeutics at Day 10 post activation. Values shown are mean \pm sem.

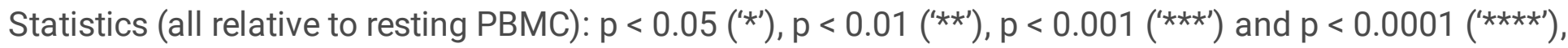
$\mathrm{n} \otimes 4$.

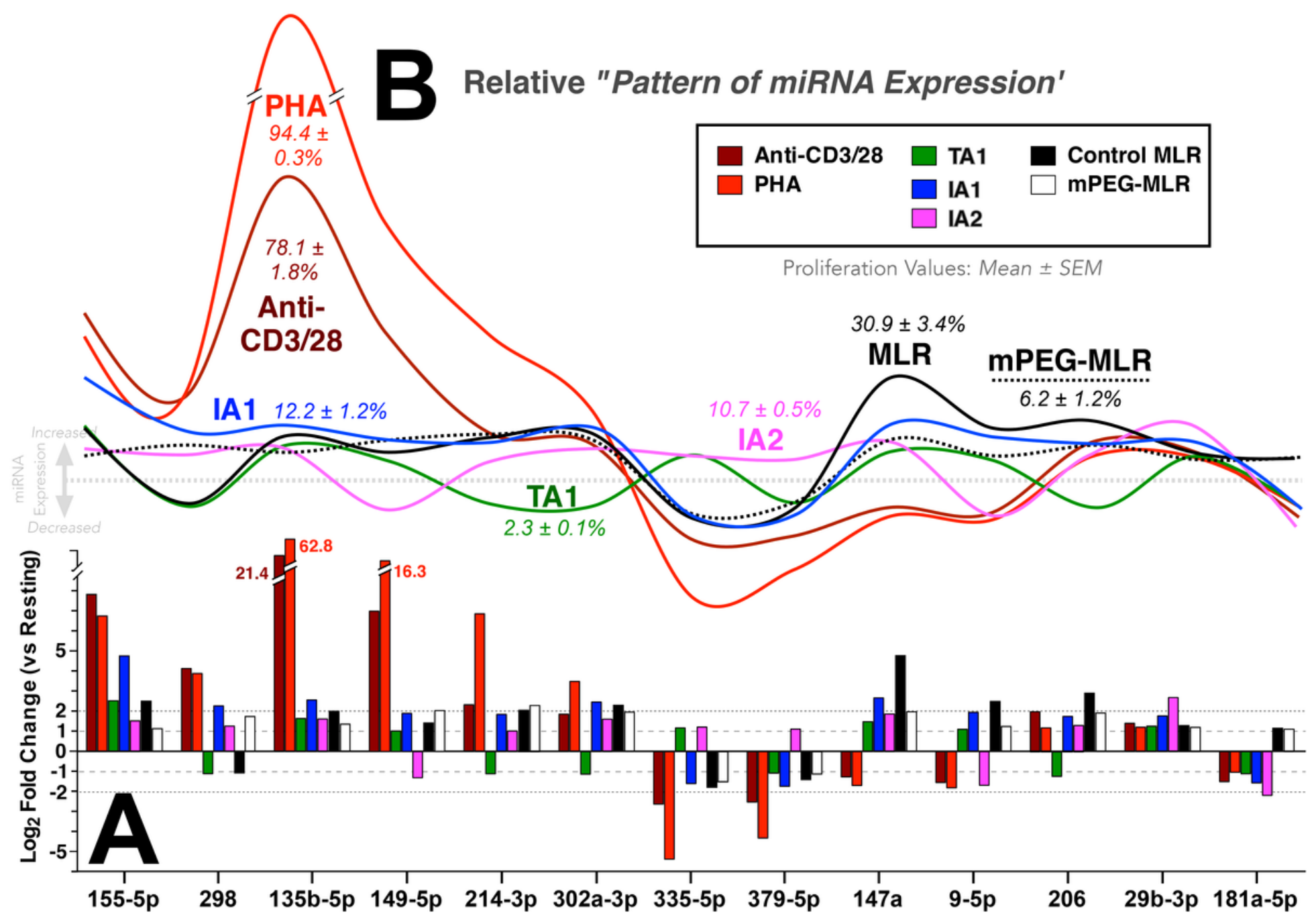

Figure 3

Significantly disparate miRNA expression patterns arise consequent to the different PBMC activation strategies. Panel A: Log2 fold change analysis similarly demonstrated the differential effects of Pan T cell activators, secretome therapeutics and allorecognition (control- and mPEG- MLR) on the expression of thirteen-selected miRNA relative to resting PBMC (solid line at 0 ). Grey dashed lines of $-2,-1,1$ and 2 values are shown for comparison purposes. Pan T cell activators altered the PBMC miRNA expression to a much larger magnitude than the pro-inflammatory IA1 secretome or the control MLR. The SYN, protolerogenic TA1 and cancer-derived IA2 secretome exerted significantly different effects on miRNA expression as would be expected based on their differential effects on cell proliferation and CD4/CD8 differentiation. Panel B: Shown are the relative 'pattern of miRNA expression' and percent proliferation of the different activation protocols from Panel A. As shown, significant differences in the pattern of 
expression are noted - even amongst the pro-inflammatory (PHA, Anti-CD3/28, IA1 and IA2) activators. The putative/described functions of these miRNA in biological studies are presented in Supplementary Table 1S.

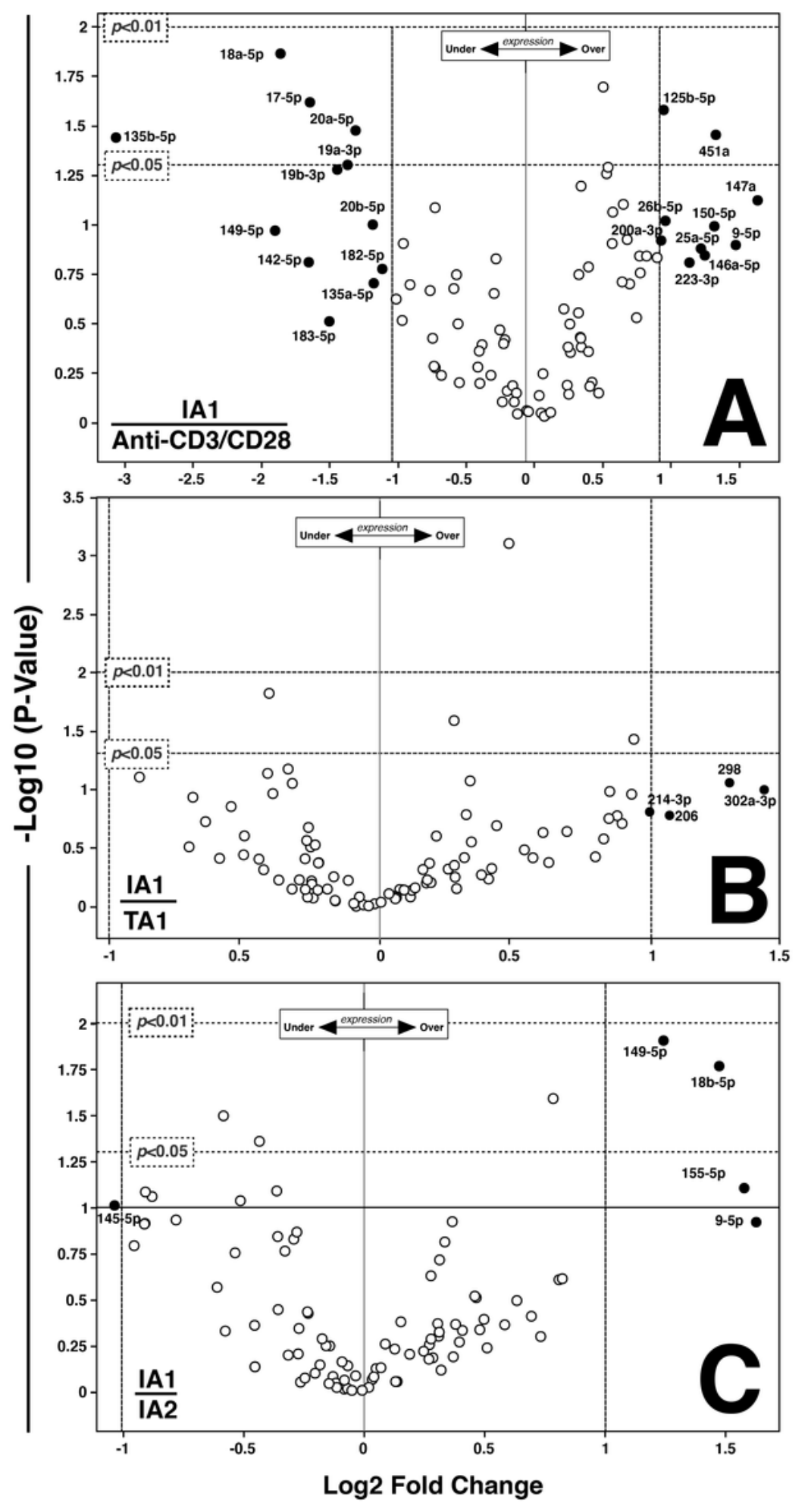

Figure 4

Volcano plot analysis of the secretome agents demonstrated differential PBMC miRNA expression profiles. Volcano plot analysis of selected secretome products was done to better compare the effects of 
these agents on resting PBMC miRNA expression 72 hours post activation.[4] Panel A: IA1 versus antiCD3/CD28. Panel B: IA1 versus TA1. Panel C: IA1 versus IA2. Vertical (Log2 Fold Change) and horizontal (-Log10; $p$ value $<0.05$ and 0.01) dashed lines denote miRNA over- or under- expressed. Unchanged (and unlabeled) miRNA are open circles while miRNA exhibiting significant changes are black circles and labeled.

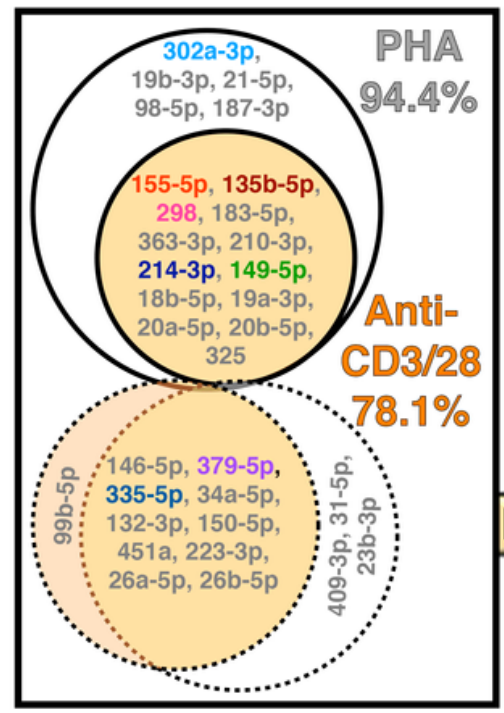

A, $\mathbf{B}$ and $\mathbf{C}$ refer to Figs 1,2,4

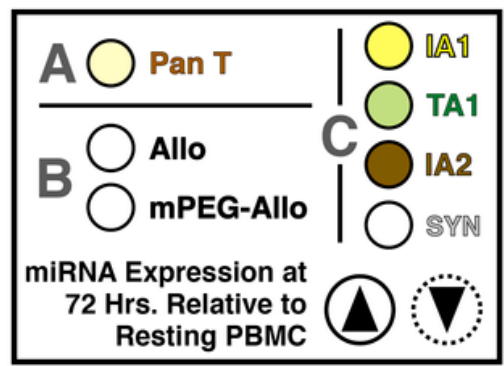

Proliferation Values: PHA, Day 4; Anti-CD3/28, Day 3; All Others Day 10

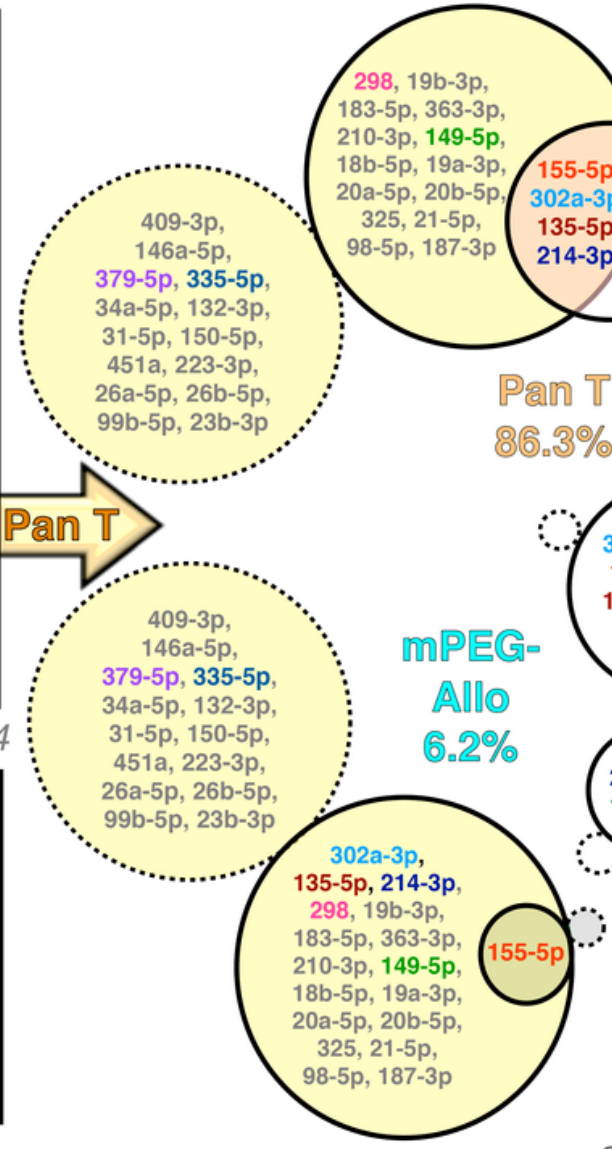

SYN 10.4\%

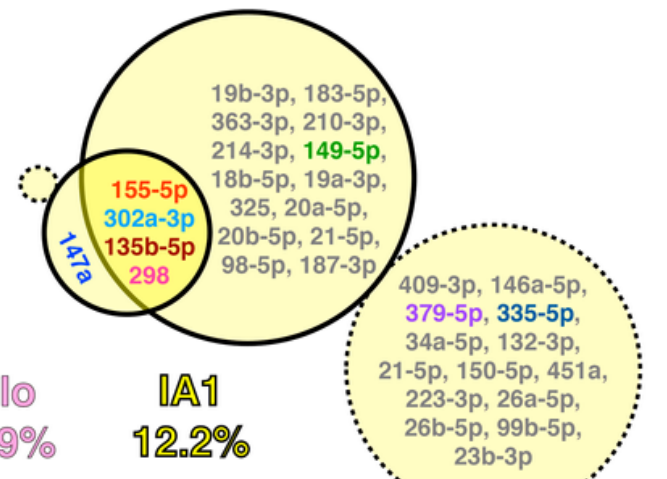

$\because \quad 23 b-3 p$
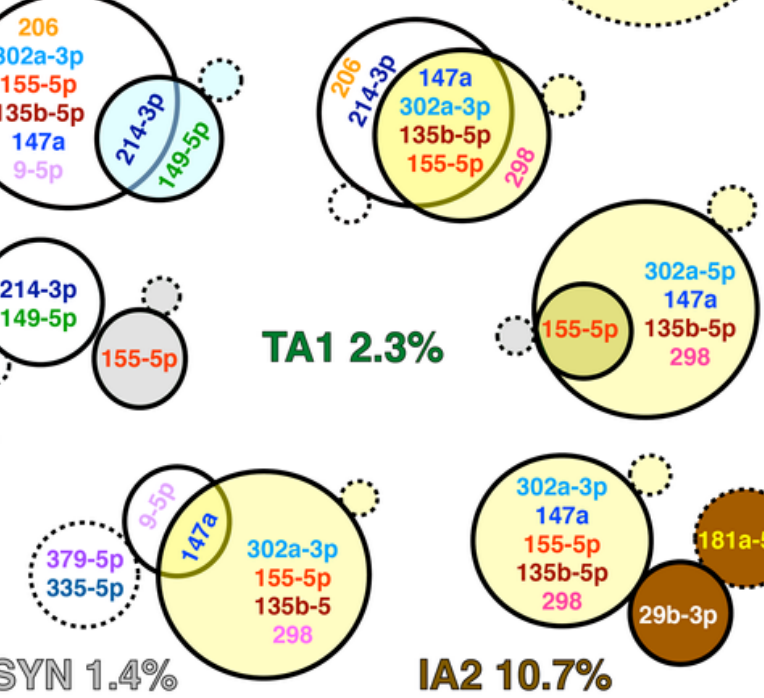

IA2 10.7\%

\section{Figure 5}

Venn diagram comparison between Pan T Cell (A), Allo (B) and Secretome (C) activation of resting human PBMC. Over-expressed miRNA are listed in solid line bubbles, while under-expressed miRNA are in dashed line bubbles. Overlapped areas indicate miRNA with similarly increased/decreased miRNA expression. The bubble color indicates different activation treatments. miRNA of interest (relative to Figures 3-4; Supplementary Table 1S) are in color. As shown, pan T cell, allo- and secretome activation exerted distinct patterns of miRNA expression in treated resting PBMC and their putative functions are described in Supplementary Table 2S. As shown, Pan T cell activators exhibit huge changes (up/down) in a large number of miRNA. In contrast, alloactivation (both the control- and mPEG-MLR) showed dramatically reduced miRNA changes and the secretome products showed an even further reduction in the differentially expressed miRNA. Proliferation values for each approach/agent are provided within the figure. 

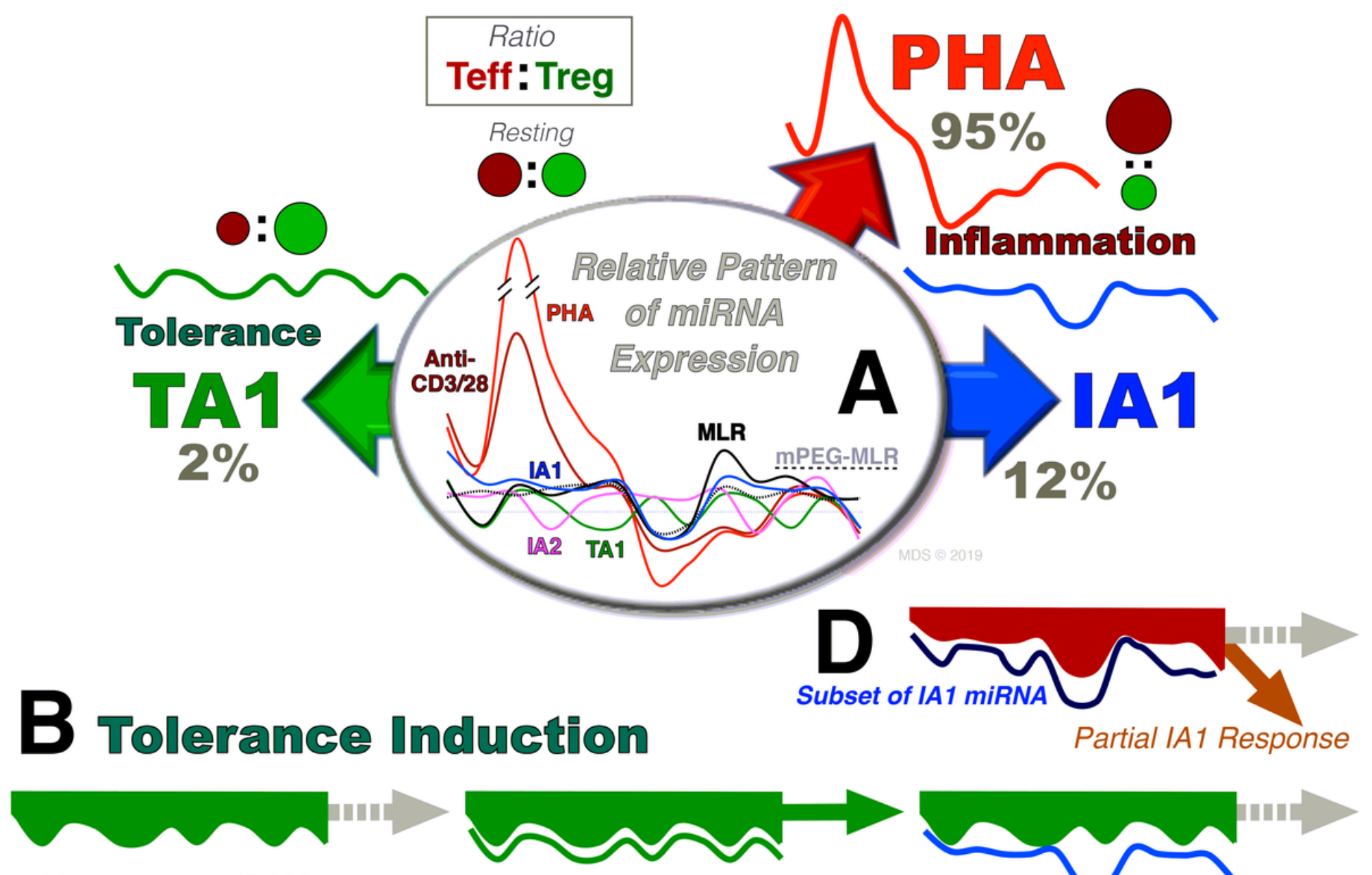

Necessary miRNA Bioregulatory Pattern TA1
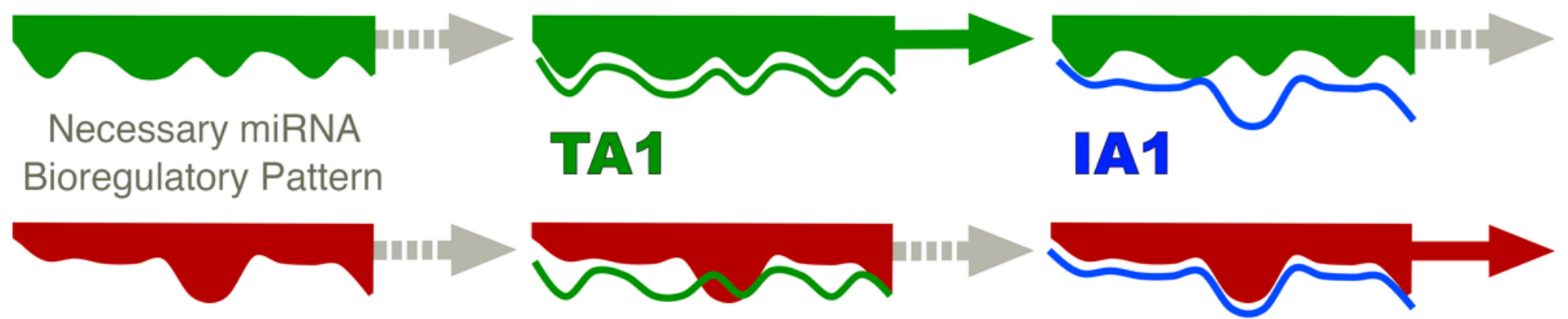

\section{C controlled Inflammation}

Figure 6

Bioregulation occurs consequent to pattern specificity of miRNA expression. miRNA are 'low fidelity' due to the promiscuous nature of miRNA: a single miRNA can interact with hundreds of genes and a single gene can interaction with multiple miRNA. Hence, the 'Pattern of miRNA' (both species and relative abundance) govern the biologic response in a 'lock and key' manner. Panel A: PBMC activation with Pan T cell, Allorecognition-driven, and secretome products yield dramatically different miRNA expression patterns and proliferation rates. Panel B-C: Using a 'lock and key' analogy, TA1 (B) and IA1 (C) induce miRNA expression profiles that induce exclusively induce either a tolerogenic (TA1) or pro-inflammatory (IA1) effect in treated PBMC. Panel D: However, if partial pattern parity exists, aspects of the biological response may be retained. As shown a similar IA1-like pattern may induce a partial response. Indeed, per Figure 6, IA1 may be viewed as a subset of the alloresponse which itself is a subset of Pan T cell 
activators. The disparity in the miRNA expression between these three pro-inflammatory responses is similarly reflected in the reduction of proliferation noted between the activators.

\section{Supplementary Files}

This is a list of supplementary files associated with this preprint. Click to download.

- zSupplementalTable2S.pdf

- zSupplementalTable1S.pdf 